\title{
Magnonic crystal with strips of magnetic nanoparticles: model- ing and experimental realization via a dip-coating technique
}

\author{
Zorayda Lazcano-Ortiz ${ }^{1}$, Cesar L. Ordóñez-Romero ${ }^{2 *}$, Jorge Luis Domínguez-Juárez ${ }^{3,4 *}$, Guillermo Monsivais ${ }^{2}$, \\ Rafael Quintero-Torres ${ }^{4}$, Daniel Matatagui ${ }^{5}$, Naser Qureshi ${ }^{6}$ and Oleg Kolokoltsev ${ }^{6}$. \\ 1 Instituto de Física, Benemérita Universidad Autónoma de Puebla, Puebla, 72570, México; \\ zlazcano@ifuap.buap.mx \\ 2 Instituto de Física, Universidad Nacional Autónoma de México, Ciudad de México 04510, México; \\ cloro@fisica.unam.mx; monsi@fisica.unam.mx \\ 3 Cátedras CONACyT, Centro de Física Aplicada y Tecnología Avanzada, Universidad Nacional Autónoma \\ de México, Juriquilla, Querétaro 76230, México; jldominguezju@conacyt.mx; \\ 4 Centro de Física Aplicada y Tecnología Avanzada, Universidad Nacional Autónoma de México, Juriquilla, \\ Querétaro, 76230, México. rquintero@fata.unam.mx; \\ 5 SENSAVAN, Instituto de Tecnologías Físicas y de la Información (ITEFI), CSIC, Serrano 144, 28006 Madrid, \\ Spain \\ 6 Instituto de Ciencias Aplicadas y Tecnología, Universidad Nacional Autónoma de México, Ciudad de \\ México 04510, México; naser.qureshi@icat.unam.mx; oleg.kolokoltsev@icat.unam.mx \\ * Correspondence: jldominguezju@conacyt.mx ; cloro@fisica.unam.mx
}

\begin{abstract}
In this article, we show theoretically and experimentally the formation of spin-waves band gaps in a magnonic crystal that was implemented by the deposition of periodic micro-structured strips of magnetite nanoparticles. A theoretical model describing the spectra of the transmitted spinwaves bandgaps is proposed. This is achieved using a simple model based on microwave transmission line theory and considering the presence of micro-structured strips of magnetite nanoparticles on the surface. Such magnonic crystal of equally spaced micro-structured strips of magnetite nanoparticles on the surface of an yttrium iron garnet thin film has been implemented and measured. The periodic micro-structured nanoparticles are deposited on the surface of such yttrium iron garnet single-crystal film grown on a gallium-gadolinium garnet substrate via dipcoating technique. Propagation of magnetostatic surface spin-waves is studied and it is shown that the presence of such periodic structure leads to the formation of spin-wave band gaps in the transmission characteristics. The spin-wave detection has been carried out using a pair of microwave antennas and a vector network analyzer. The results show that the periodic structure formed by the magnetite strips modifies the spectra of the transmitted spin waves producing band gaps.
\end{abstract}

Keywords: magnonic crystal; spin waves; magnetite nanoparticles.

\section{Introduction}

Magnonic crystals (MCs) and the way the spin-waves behave inside them have attracted significant attention in recent years. [1-5]. The different techniques to produce such magnonic devices have been based on, chemical etching, metal deposition, and ion implantation, and many other methods that can introduce a periodic variation of any magnetic parameter [6-14]. Similar to their phononic and photonic counterparts, the presence of a periodic structure in MCs leads to the formation of artificially tailored band gaps, wherein spin-wave propagation is not allowed. The formation of MCs by a periodic variation in the local temperature at the surface of a ferromagnetic film has also been demonstrated [15]. Up to now, the most studied MC is built from a thin yttrium iron garnet (YIG) films with a chemical-etched array of parallel grooves on the surface has been the most popular media to implement the MCs due to their special characteristics and performance [9-11]. The theoretical investigation and experimental observation of band 
gaps have been reported including current-controlled magnonic crystals $[8,16]$, bicomponent one-dimensional magnonic crystals of different magnetic materials [17], patterning the magnetic properties by localized ion implantation [18], planar magnonic crystals with different kinds of overlayers [19-21] or as we show here, by deposition of periodic micro-structured strips of magnetite nanoparticles.

It has been demonstrated that the spin waves are very sensitive to the boundary conditions of the interfaces of the magnonic waveguides [19,22]. Recently, alternative devices such as a magnetic gas sensor based on the combination of magnetic nanoparticles $\left(\mathrm{CuFe}_{2} \mathrm{O}_{4}\right)$ deposited on YIG films have been also demonstrated [23,24]. These magnonic gas sensors take advantage of the high sensitivity of magnetostatic spin-waves to changes induced in the boundary conditions due to the magnetic properties of nanoparticles interacting with gases. In Ref. [23] and [24], it is shown that the magnetic nanoparticles can detect low concentrations of different volatile organic compounds at room temperature, with high sensitivity, short response time, and good reproducibility. On the other hand, the formation of a band structure of spin waves in MCs, and the possibility of tuning frequency, width, and depth of these bandgaps, allowing a resonant behavior, enabling a set of potential applications mixing the nature of periodic structures with sensitive magnetic nanoparticles. The technological developments of MC devices with magnetic nanoparticles could be an essential tool for spin-waves research, such as bandpass or notch filters, single-mode waveguides that can increase the transmission efficiency, or bending waveguides with suppressed energy leakage, similar to what has been obtained in photonic waveguides [25].

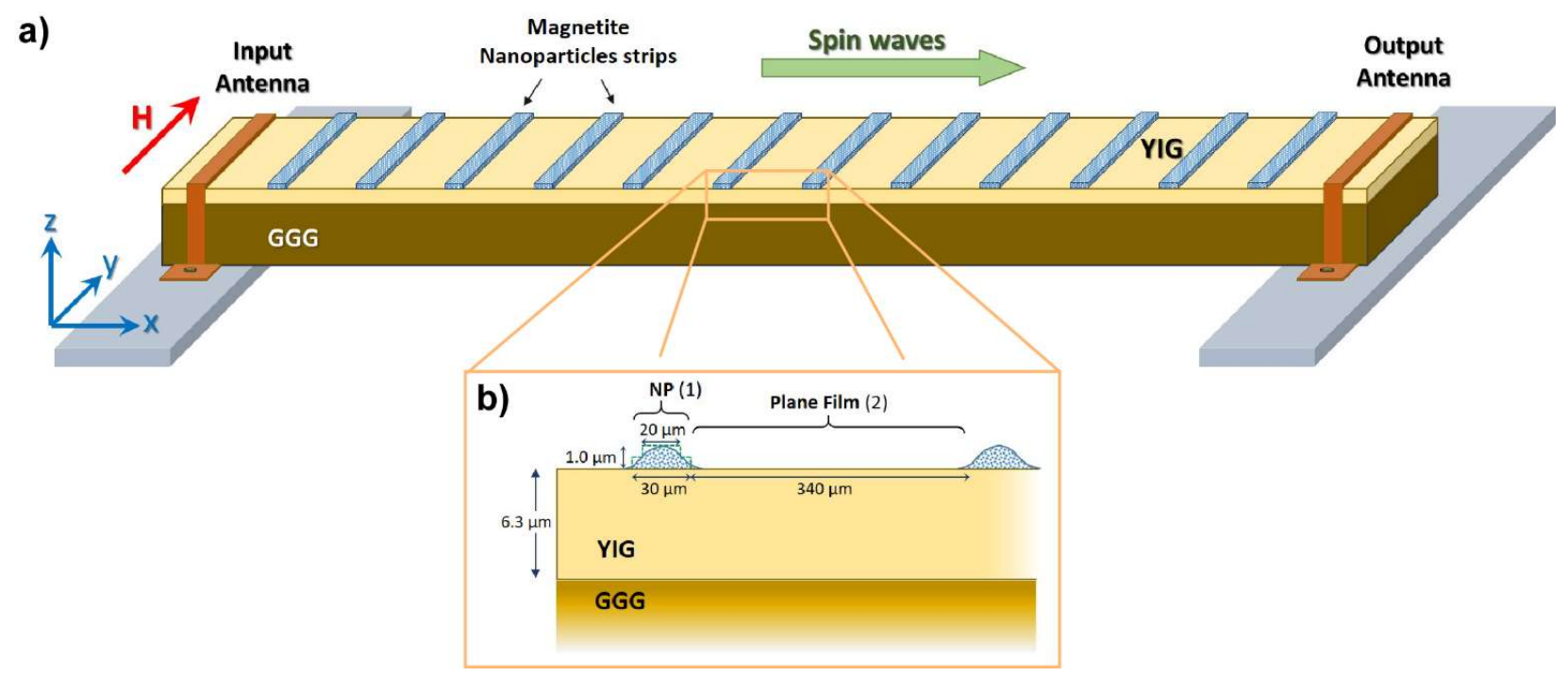

Figure 1. (a) Schematic representation of the experimental setup used to measure MSSW transmission. (b) Schematic profile view of one side period of a magnonic crystal by deposition of magnetite nanoparticles via dip coating on a YIG films sample. Blue dotted areas correspond to the approximate profile of the deposited nanoparticles strips. The dashed line shows the profile used for calculations. The thickness and the length (not in scale) of each region are shown.

In this Letter, we theoretically modeled and experimentally measured the formation of a band structure for spin-waves in MCs by deposition of periodic micro-structured magnetite nanoparticles (NPs) via dip coating. The theoretical model describes how the periodic structure leads to the formation of spin-wave band gaps. The spin-wave transmission through the MC is described in terms of the transfer matrix method. The sample has been treated as a periodic approximation sequence of microwave transmission 
strips surrounded by different environments due to the presence of the periodic microstructured NPs that modify the conditions of propagation. Access to the band gaps for spin-waves in MC is possible with NPs. The formation of the spin-wave bandgaps is experimentally demonstrated by periodic micro-structured magnetite nanoparticles on a YIG film. The MC presented here offers several advantages as it is obtained with microstructured magnetite nanoparticles via dip coating on a YIG film, allowing the observation of spin-wave band gaps and providing an alternative tool in the fabrication of MCs.

\section{Results}

\subsection{Theoretical model}

Following the schematics in Figure 1, the MC studied here consists of a pattern of equally spaced magnetite NPs strips perpendicular to the spin-wave propagation direction. The theoretical model proposed to calculate the spin-wave transmission spectrum treats the scattering of the magnonic crystal considering the patterned structure as a periodic sequence of microwave transmission strips with different propagation constants for the same transmitted frequency [11].

The spin-wave transmission through the MC is described in terms of a transmission matrix in each section of the MC considering the interference effects simplistically. Each period of the MC consists of two sections indicated in Figure 1b: (1) a region with magnetite NPs deposited on the surface of YIG film and (2) a section of plane YIG film (Plane Film-PF) with only air on the surface. The model considers that the presence of NPs on the surface of the YIG film modifies the propagation of spin waves in the region (1).

The matrices that describe the spin-wave propagation either in the PF regions or in the NP regions are given by:

$$
T_{j}=\left(\begin{array}{cc}
e^{-\left(i k_{j}-k_{j}^{\prime}\right) L_{j}} & 0 \\
0 & e^{\left(i k_{j}-k_{j}^{\prime}\right) L_{j}}
\end{array}\right)
$$

here, $\mathrm{j}$ could be either NP or PF, $L_{j}$ is the length of the corresponding region and, $k_{j}$ and $k_{j}^{\prime}$ are the spin-wave wavenumber and the spatial damping rate, respectively. The spinwave wavenumber for MSSW is calculated using [40]:

$$
k_{j}=-\frac{1}{2 d} \ln \left\{1+\frac{4}{\omega_{M_{j}}{ }^{2}}\left[\omega_{0}\left(\omega_{0}+\omega_{M_{j}}\right)-\omega^{2}\right]\right\},
$$

where $k_{N P}$ and $k_{P F}$ will have different values in each section of the MC and depend on $\omega_{M_{j}}$. For PF regions, $\omega_{M_{P F}}=2 \pi|\gamma| M_{s}$, where Ms is the saturation magnetization of YIG and $\gamma$ the gyromagnetic ratio. For NP regions, $\omega_{M_{N P, i}}=2 \pi|\gamma| M_{s} \xi_{i}$, where the parameter $\xi_{i}$ is introduced to describe the changes coming from the interaction between YIG film and the deposited magnetite NPs. Sub-index $i$ is used to distinguish the two steps of the dashed strip in Figure $1 \mathrm{~b}, i=1$ to the first step and $i=2$ to the second step. The thickness of the plane YIG film is given by $d$ and $\omega_{0}=2 \pi|\gamma| H_{0}$, where $H_{0}$ is the applied magnetic bias field.

To calculate the spatial damping rate in each region, we consider the spin-wave loss as due to intrinsic magnetic damping only, which give us: $k_{j}^{\prime}=2 \pi|\gamma| \Delta H \zeta_{j} /\left|v_{g_{j}}\right|$, where $\Delta \mathrm{H}$ is the ferromagnetic resonance half-power linewidth. The spin-wave group velocity, $v_{g_{j}}=\partial \omega / \partial k_{j}$, for MSSW can be calculated as in [26], and regions are given by: 


$$
v_{g_{j}}=\frac{\omega_{M_{j}}^{2} d_{j}}{4 \omega} e^{-2 k_{j} d_{j}}
$$

The parameter $\zeta_{j}$ is introduced to consider the losses due to the presence of magnetite NPs on the surface. For PF regions $\zeta_{P F}=1$, and for NP regions $\zeta_{N P}>1$.

Since the presence of NPs modifies the conditions of propagation, we consider that the spin waves will be partially reflected when they go through the interface between the different regions. This condition of reflection at the edges of the regions has to be taken into account and can be represented with two additional matrices with the form:

$$
T_{v}=\left(\begin{array}{cc}
(1-v \Gamma)^{-1} & v \Gamma(1-v \Gamma)^{-1} \\
v \Gamma(1-v \Gamma)^{-1} & (1-v \Gamma)^{-1}
\end{array}\right),
$$

where $\Gamma$ is the reflectivity and $v$ is the direction index: $v=+1$ is used for the edge where the spin-wave is coming from the PF section of the film towards the NP strip, and $v=-1$ for the edge where the wave is coming from the NP strip towards the PF section.

Meanwhile, the structure has been treated as a periodic sequence of microwave transmission strips surrounded by different environments. It is reasonable to define $\Gamma$ in terms of a difference in the "characteristic impedance" of the YIG film in the different regions. Assuming the influence of the material deposited on the surface of the YIG film can be considered a variation of the film's effective inductance, $\Gamma$ can be written as: $\Gamma=$ $\left(k_{N P}-k_{P F}\right) /\left(k_{N P}+k_{P F}\right)$.

Finally, using the transmission matrix properties, the propagation through a complete period could be obtained by multiplying the matrices, $T=\left[T_{P F} \cdot T_{+} \cdot T_{N P} \cdot T_{-}\right]$, and, for the complete MC with $N$ periods, the total matrix is given by the product of $N$ transfer matrices $T$. The matrix $T_{N P}$ contains the information about the two steps necessary for the computation analysis of NPs regions (as is shown by the green dashed line in Figure $1 \mathrm{~b}$ ). Then $T_{N P}=T_{N P, 1} \cdot T_{+} \cdot T_{N P, 2} \cdot T_{+} \cdot T_{N P, 1}$, where $T_{N P, 1}$ is the matrix associated with the first step of length $L_{j}=L_{N P, 1}$ and $T_{N P, 2}$ is the matrix associated with the second step of length $L_{j}=L_{N P, 2}$. Finally, the power transmission coefficient of the magnonic crystal can be determined as $P_{t r}=1 /\left|T_{11}^{M C}\right|^{2}=1 /\left|T_{22}^{M C}\right|^{2}[11]$.

\subsection{Experiment}

Figure 1 shows a schematic view of the delay structure used to measure the amplitude-frequency characteristic of the magnonic crystal. For this configuration magnetostatic surface spin waves (MSSW) are excited. The static external field $H$ was applied parallel to the surface of the YIG film ( $y$-axis) and perpendicular to the spin-wave propagation direction ( $x$-axis). The field was set up at a nominal value of 770 Oe. To excite (input) and detect (output) the spin waves, a pair of $20 \mu \mathrm{m}$-diameter gold wire microwave antennas were placed directly on the surface of the YIG film with a separation of $6 \mathrm{~mm}$ so that the patterned array of NPs was entirely localized between them. A vector network analyzer connected to these antennas was used to measure the amplitude-frequency characteristic of the magnonic crystal.

The amplitude-frequency characteristic was measured using a vector network analyzer connected to the input and output antennas. Figure 2a presents, on a logarithmic scale, the transmission spectra of MSSW measured for a completely planar YIG film (blue thin line), and the periodic structured sample described in the Materials and Methods section (black thick line). In Figure 2b, both the experimental (black thick line) and the theoretically simulated (red thin line) amplitude-frequency characteristic for the fabricated MC are shown. The red thin line corresponds to the theoretical prediction of the transmission spectrum based on the theoretical model described in the next Materials and Methods section while the black thick line corresponds to the measured spectrum. The 
values used for the simulation are: $N=12, d=6.3 \mu \mathrm{m}, L_{P F}=340 \mu \mathrm{m}, L_{N P, 1}=5 \mu \mathrm{m}$ and $L_{N P, 2}=20 \mu \mathrm{m}$, in such a way that $L_{N P}=L_{N P, 1}+L_{N P, 2}+L_{N P, 1}=30 \mu \mathrm{m}$, as is shown in Figure $1 \mathrm{~b}$. The best fit corresponds to the parameters $\xi_{1}=0.96, \xi_{2}=0.94$ and $\zeta_{N P}=3.0$.

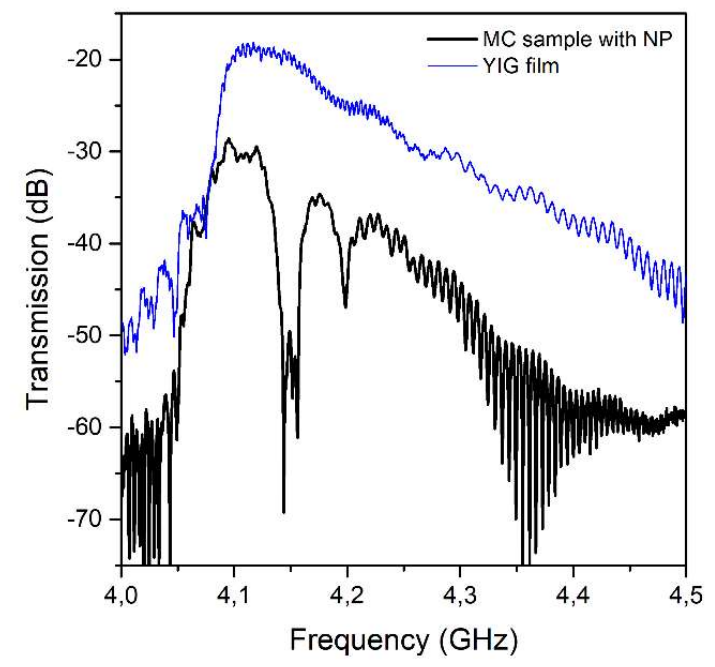

(a)



(b)

Figure 2. (a) Experimental signature of the spin-wave band gaps in the magnonic crystal with magnetic nanoparticles. The thin line (blue) corresponds to the spin-wave transmission spectrum of the YIG film (before nanoparticles deposition), and the thicker line (black) corresponds to the signal of the structured film, i.e. the magnonic crystal. (b) Theoretical results for the transmission spectrum (red thin line) are plotted to compare with the experimental measurement (black thick line). Both figures are plotted using a logarithmic scale for the $y$-axis.

\section{Discussion}

From Figure 2a, the effect of the equally spaced NP strips deposited on the surface of the YIG film is shown (black thick line). We observe two regions with important losses at certain frequencies: about $20 \mathrm{~dB}$ at $4.15 \mathrm{GHz}$ and $10 \mathrm{~dB}$ around $4.20 \mathrm{GHz}$. These drops in the transmission spectrum correspond to spin waves band gaps. We observed at $4.35 \mathrm{GHz}$ another region that could be seen as a bandgap, however, this behavior is more likely attributed to the shrinking of the amplitude-frequency characteristic because of the presence of an additional noninterference damping mechanism. The presence of the characteristic oscillations in the amplitude denotes the interference of the spin waves with the electromagnetic waves propagated through the air between the antennas besides the smooth and systematic decrease of transmission intensity for all frequencies. The periodic structure modifies the spin-wave transmission. Previously, it has also been demonstrated that periodic systems, such as photonic and phononic crystals can be used as sensors due to their well-defined physical properties such as reflectance/transmittance, resulting in higher levels of sensitivity [27-31]. The presence of photonic or phononic band gaps (regions of frequency where light or sound are forbidden to propagate) enables the control of light (or sound) with remarkable facility and produces effects that are not possible with non-structured systems [27]. It has been demonstrated that these band gaps are strongly dependent on the properties of molecules that interact with the crystal, thus the penetration of molecules within the structure modifies spectral position, intensity, and width of the band gaps [29]. In this work, the periodic structure of the magnetite nanoparticles modifies the spin-wave transmission and generates the MC. The positions of the band 
gaps of the system are expected to be very sensitive to the environment surrounding the MC. In this way, it is evident that the design of an MC with a periodic structure of magnetic nanoparticles could act as a highly sensitive device that takes advantage of the characteristic resonant capabilities of the structured media. Our model (red thin line in Figure $2 \mathrm{~b})$ predicts five-band gaps in the observed frequency range $(4.12-4.32 \mathrm{GHz})$. This is due, among other things, to the fact that our model considers a perfect periodic system, with parallel and well-defined nanoparticles strips, while the experiment only occurs at the center of the YIG film. With the simple dip-coating technique presented here, we experimentally observed the first two band gaps with high resolution and the last bandgaps require more perfect strips. In Figure 3b, it can be observed that the nanoparticle strips in the sample are equally spaced, but they are not straight micro-structures nor perfectly defined close to the borders. Despite these differences, the position of the first bandgaps is well predicted.

We experimentally show that the dip-coating technique can be considered as an alternative tool in the fabrication of MCs, mainly because it has the micrometric resolution needed for this kind of structure [32]. Dip-coated deposition depends on interface and parameters used in the fabrication process, such as substrate temperature, solvent or composition of the nanofluid, and finally the shape of the nanostructures [33]. This fabrication method of magnonic structures has multiple advantages, which are led by the possibility of inducing significant changes in the amplitude-frequency characteristic of the magnonic waveguides. The design of magnonic periodic structures with a wide range of different sizes, shapes, and properties of magnetite nanoparticles could be used to develop highquality resonant sensors with the extraordinary capability to reuse the YIG strip by removing the nanoparticles from its surface without producing any permanent changes. The possible application of MCs as components for the processing of analog and digital information [34]. Furthermore, since MCs are sensitive to the external magnetic field, they have been demonstrated as extremely sensitive magnetic field sensors at room temperature [35]. The MCs presented here are of great interest for research from a wide range of fields, including magnetic fluids, catalysis, biotechnology, biomedicine, biosensing applications, magnetic resonance imaging, data storage, and environmental remediation [36]. The applications in biomedicine stand out and include tumor tissue targeting, local hyperthermia effect, drug delivery, and magnetic resonance imaging diagnostics [37]. The great potential for use in medicine is due to their biocompatibility, biodegradability, easy synthesis, and ease with which they may be tuned and functionalized for specific applications [38].

\section{Materials and Methods}

The magnonic structure was fabricated using a uniform long and narrow YIG film strip epitaxially grown in the (111) crystallographic plane on a gallium gadolinium garnet (GGG) substrate of thickness $500 \mu \mathrm{m}$. The YIG strip was 35mm long, $1.7 \mathrm{~mm}$ wide, and $6.3 \mu \mathrm{m}$ thick. The high quality of the YIG film is highlighted by the ferromagnetic resonance half-power line width $(\Delta \mathrm{H})$ which is about $0.5 \mathrm{Oe}$ at $5 \mathrm{GHz}$. The patterned structure was carried out by controlled deposition on magnetite nanoparticles on the sample surface quality via dip coating. Commercial magnetite nanoparticles with a diameter of $30 \pm$ $2 \mathrm{~nm}$ were used (777408, Sigma Aldrich). Figure 3a shows the experimental and schematic view of the setup mounted to deposit NPs strips on YIG film. A magnetite solution of $8 \times 10^{-4} \%$ weight per volume diluted in 1-propanol was sonicated for one hour to minimize particle aggregation [39]. For homogeneity and reproducibility, a motorized stage was used for controlling a regular immersion and retrieval, while the choice of solvent enhanced wetting and evaporation in the meniscus region [40]. To obtain the gratings, the translation stage in the immersion was scanned to deposit a series of parallel stripes, that comes from the meniscus of the magnetite solution of approximately 30 microns wide and less than 1.7 millimeters (the wide of the YIG film), leaving in between a non-excess stripe 
of the 340 widths. The period was $370 \mu \mathrm{m}$ (30/340) $30 \mu \mathrm{m}$ a region with magnetite nanoparticles (NP) deposited on the surface of YIG film and $340 \mu \mathrm{m}$, a section of plane YIG film. The motorized stage was moved with different displacements steps corresponding to the 30/340 period. The $30 \mu \mathrm{m}$ region with NPs was created with six displacement steps of $5 \mu \mathrm{m}$, run at a stage speed of $0.01 \mathrm{~mm} \mathrm{~s}^{-1}$ and each step with a dwell time of one minute. To the $340 \mu \mathrm{m}$ section, the motorized stage was moved using one step, run at a stage speed of $1 \mathrm{~mm} \mathrm{~s}^{-1}$. Figure $3 \mathrm{~b}$ presents an image of the top view of a sample fabricated in this way. One side of the substrate was carefully cleaned, leaving the strips of the nanoparticles only on one side. From Figure $3 b$ the NPs strips are approximately straight in the middle of the YIG film and there is a discontinuous of such strip close to the lateral borders. Such discontinuous phenomena are due to the surface tension of the dip-coating solvent in a combination of the inhomogeneities of the border at the YIG waveguide surface. The patterned array characterized here corresponds to twelve NPs strips, meaning the MC with a lattice constant of $370 \mu \mathrm{m}$.
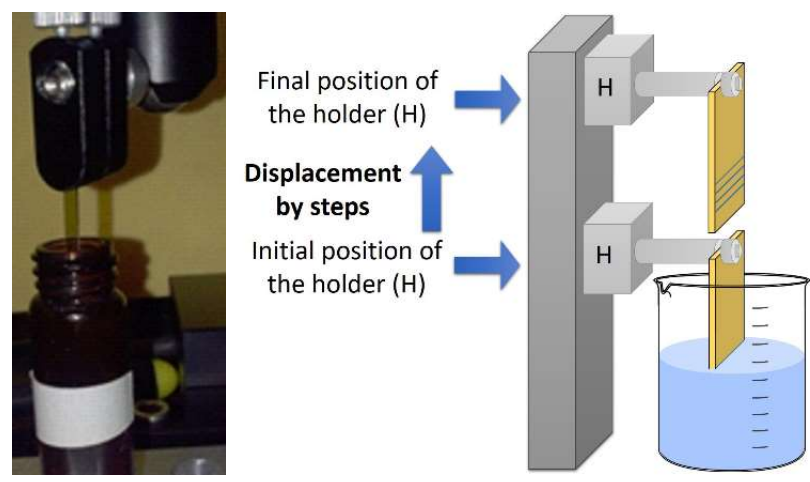

(a)

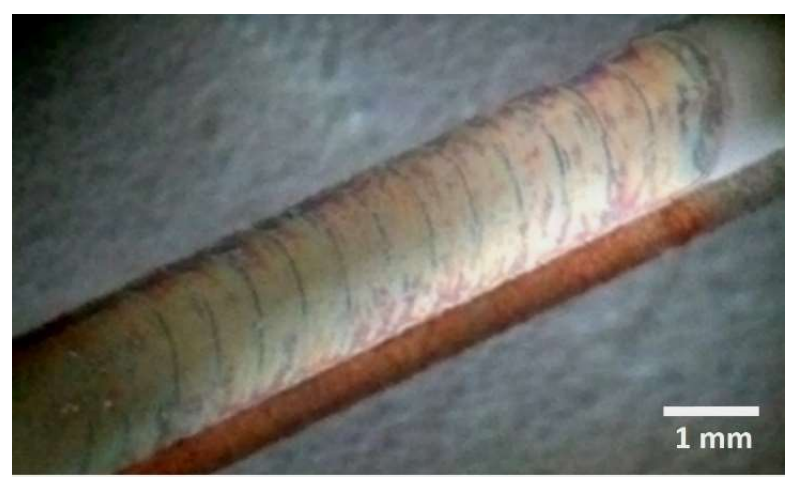

(b)

Figure 3. (a) Experimental and schematic view of the process for depositing a periodic nanoparticle pattern on the YIG waveguide surface via the deep coating technique. The first strip of NPs is deposited when the holder is at its initial position and as it goes up the subsequent strips are deposited. (b) Image of a sample fabricated by using the experimental process in (a).

\section{Conclusions}

We modeled and experimentally measured band gaps of spin-wave transmission through the MC using magnetic nanoparticles. The theoretical prediction of the transmission spectrum agrees with the experimental measurement. The proposed model not only predicts the formation of band gaps in this kind of magnonic crystal, but also the formation of the MC was demonstrated by deposition of periodic micro-structured magnetite nanoparticles (NPs) via dip coating on the YIG waveguide surface. The theoretical results are in good agreement with the experimental data since our model correctly predicts the formation of band gaps for MSSW. It has been demonstrated that the experimental dip-coating deposition used here is an effective technique to fabricate MCs. Although the process can be improved, this technique is enough to observe the gaps and offers several advantages. It produces MCs easier than other common techniques, using only the periodic deposition of magnetic nanoparticles. Possible application in two-dimensional patterning enables the implementation of detection changes in transmission band gaps transmission because of the interaction with the nanoparticles. These MCs are expected to excel as sensors showing higher sensitivity than unstructured systems based on the advantage of the characteristic resonant behavior of structured media. 


\section{Patents}

This section is not mandatory but may be added if there are patents resulting from the work reported in this manuscript.

Supplementary Materials: The following are available online at www.mdpi.com/xxx/s1, Figure S1: title, Table S1: title, Video S1: title.

Author Contributions: C.L.O.R. and J.L.D.J., conceived the project; C.L.O.-R. and J.L.D.J., designed and implemented the experimental setup, and methodology; software, Z.L.O., and C.L.O.-R.; Z.L.O., C.L.O.-R., and J.L.D.J., contributed extensively to the planning and discussion of this work; validation, Z.L.O, C.L.O.R., J.L.D.-J., R.Q.-T, G.M., D.M., N.Q., and O.K.; formal analysis, Z.L.O., C.L.O.R., J.L.D.-J., R.Q.-T, G.M., D. M., N. Q., and O.K; investigation Z.L.O., C.L.O.R., J.L.D.-J, G.M., D.M., N.Q., and O.K and R.Q.-T.; resources, Z.L.O., C.L.O.R., J.L.D.-J., R.Q.-T; data curation, Z.L.O., C.L.O.R. and R.Q.-T.; writing-original draft preparation, Z.L.O., and J.L.D.-J.; writing - review and editing, Z.L.O, C.L.O.R., J.L.D.-J., R.Q.-T, G.M., D.M., N.Q., and O.K..; visualization, Z.L.O, C.L.O.R., J.L.D.-J., R.Q.-T, G.M., D.M., N.Q., and O.K.; supervision, Z.L.O, C.L.O.R., and J.L.D.-J.; project administration, Z.L.O, C.L.O.R., and J.L.D.-J.; funding acquisition, , Z.L.O, C.L.O.R., G.M. and J.L.D.$\mathrm{J}$. All authors have read and agreed to the published version of the manuscript.

Funding: The work presented here has been supported by the program CATEDRAS-CONACYT, grant number 1039 and by UNAM-DGAPA grant number AG100521. R.Q.-T. thanks to the financial support by the program PAPIME, grant number PE101319. R.Q.-T and JLDJ wish to thank the financial support from CONACYT, under grant A1-S-8317. D.M.C. wish to thank the Ministry of Science and Innovation (Spain) through the project RTI2018-095856-B-C22 (AEI/FEDER).

Institutional Review Board Statement: Not applicable.

Informed Consent Statement: Not applicable.

Data Availability Statement: Data is contained within the article or supplementary material, correspondence and requests for materials should be addressed to J.L.D.-J. (jldominguezju@conacyt.mx).

Acknowledgments: Special thanks should be given to Dr. Jose Luis Aragon Vera as a research project supervisor of CATEDRAS-CONACYT grant number 1039 in the Centro de Física Aplicada y Tecnología Avanzada at the Universidad Nacional Autónoma de México. D. M. acknowledges the financial support from the Fundación General CSIC via Programa ComFuturo.

Conflicts of Interest: The authors declare no conflict of interest.

\section{References}

1. A. B. Ustinov, N. Yu. Grigor'eva and B. A. Kalinikos, JETP Letters 2008, 88, 31.

2. A. B. Ustinov, B. A. Kalinikos, V. E. Demidov, and S. O. Demokritov, Phys. Rev. B 2010, 81, 180406(R).

3. A. V. Drozdovskii and B. A. Kalinikos, JETP Letters 2012, 95, 357.

4. A. B. Ustinov, A. V. Drozdovskii and B. A. Kalinikos, Appl. Phys. Lett. 2010, 96, 142513.

5. A. V. Chumak, V. S. Tiberkevich, A. D. Karenowska, A. A. Serga, J. F. Gregg, A. N. Slavin and B. Hillebrands, Nature Communications 2010, 1, 141.

6. A. D. Karenowska, A. V. Chumak, A. A. Serga, J. F. Gregg, and B. Hillebrands, Appl. Phys. Lett. 96, 082505 (2010)

7. Yu. V. Gulyaev and S. A. Nikitov, Dokl. Phys. 2001, 46, 687.

8. A. V. Chumak, T. Neumann, A. A. Serga, B. Hillebrands, and M. P. Kostylev, J. Phys. D: Appl. Phys. 2009, $42,205005$.

9. A. V. Chumak, A. A. Serga, B. Hillebrands, and M. P. Kostylev, Appl. Phys. Lett. 2008, 93, 022508.

10. A. V. Chumak, A. A. Serga, S. Wolff, B. Hillebrands, and M. P. Kostylev, Appl. Phys. Lett. 2009, 94, 172511.

11. A. V. Chumak, A. A. Serga, S. Wolff, B. Hillebrands, and M. P. Kostylev, J. Appl. Phys. 2009, 105, 083906.

12. F. Ciubotaru, A. V. Chumak, B. Obry, A. A. Serga, B. Hillebrands. Phys. Rev. B. 2013, 88, 134406.

13. A. V. Chumak, A. A. Serga, and B. Hillebrands, Nat. Commun. 2014, 5, 4700.

14. A. A Serga, A. V. Chumak, and B. Hillebrands, J. Phys. D: Appl. Phys. 2010, 43, 264002

15. M. Vogel, A. V. Chumak, E. H. Waller, T. Langner, V. I. Vasyuchka, B. Hillebrands, and G. von Freymann, Nature Physics 2015, 11, 487-491.

16. Q. Wang, A. V. Chumak, L. Jin, H. Zhang, B. Hillebrands, and Z. Zhong, Phys. Rev. B 2017, 95, 134433.

17. Z. K. Wang, V. L. Zhang, H. S. Lim, S. C. Ng, M. H. Kuok, S. Jain, and A. O. Adeyeye, Appl. Phys. Lett. $2009,94,083112$.

18. B. Obry, P. Pirro, T. Brächer, A. V. Chumak, J. Osten, F. Ciubotaru, A. A. Serga, J. Fassbender, and B. Hillebrands, Appl. Phys. Lett. 2013, 102, 202403.

19. M. L. Sokolovskyy, J. W. Klos, S. Mamica, and M. Krawczyk, J. Appl. Phys. 2012, 111, 07 C515. 
20. A. A. Nikitin, A. A. Nikitin, A. B. Ustinov, E. Lähderanta and B. A. Kalinikos, IEEE Trans. Mag., 2018, 51(11), 2501805

21. A. B. Ustinov, A. V. Drozdovskii, A. A. Nikitin, A. A. Semenov, D. A. Bozhko, A. A. Serga, B. Hillebrands, E. Lähderantaand B. A. Kalinikos, Commun. Phys. 2019, 2, 137.

22. N. Y. Grigorieva, and B. A. Kalinikos, Tech. Phys. 2009, 54, 1196.

23. D. Matatagui, O. V. Kolokoltsev, N. Qureshi, E. V. Mejía-Uriarte, J.M. Saniger , Nanoscale 2015, 7, 9607-9613.

24. D. Matatagui, O. V. Kolokoltsev, N. Qureshi, E. V. Mejía-Uriarte, C. L. Ordoñez-Romero, A. Vázquez-Olmos, J. M. Saniger, Sensors and Actuators B 240 (2017) 497-502.

25. M. Krawczyk and D. Grundler, J. Phys.: Condens. Matter 2014, 26, 123202.

26. D. D. Stancil and A. Prabhakar, Spin Waves: Theory and Applications, Springer, New York, USA 2009.

27. H. Xu, P. Wu, C. Zhu, A. Elbaz and Z. Z. Gu, J. Mater. Chem. C, 2013,1, 6087-6098.

28. A. M. Ahmed and A. Mehaney, Scientific Reports 2019, 9, 6973.

29. I. I. Ivanov, V. A. Skryshevsky, A. Belarouci, Sensors and Actuators A 2020, 315, 112234.

30. R. Lucklum, M. Zubtsov and M. Ke, IEEE Transactions on Ultrasonics, Ferroelectrics, and Frequency Control 2012, 59, 3.

31. S. M. Shaban, A. Mehaney, and A. H. Aly, Appl. Opt. 2020, 59, 3878-3885.

32. J. L. Domínguez-Juárez, C. L. Ordóñez-Romero, D. Matatagui, O. Kolokoltsev, R. Quintero-Torres, J. L. Aragón, (2015, August 16-20) [Oral Presentation] Magnonic crystal via dip coating nanoparticles films, XXIV International Materials Research Congress, Cancun, Mexico.

33. Y. Wang, M. Zhang, Y. Lai, L. Chi. Advanced colloidal lithography: From patterning to applications, Nano Today 2018, 22, 3661.

34. A. V. Chumak, A. A. Serga, and B. Hillebrands, J. Phys. D: Appl. Phys. 2017, 50, 244001

35. M. Inoue, A. Baryshev, H. Takagi, P. B. Lim, K. Hatafuku, J. Noda, and K. Togo, Appl. Phys. Lett. 2011, 98, 132511.

36. A. Lu, E. Salabas, and F. Schüth, Angew. Chem. Int. Ed. 2007, 46, 1222 - 1244. [

37. C. Alexiou, R. J. Schmid, R. Jurgons, M. Kremer, G. Wanner, C. Bergemann, E. Huenges, T. Nawroth, W. Arnold, and F. G. Parak, Eur Biophys J 2006, 35: 446.

38. R. A. Revia, M. Zhang, Materials Today 2016, 19 (3), 157-168.

39. X. Haitian, J. Steven, C. Byoung-Chul, G. Reuven. Characterization of Individual Magnetic Nanoparticles in Solution by Double Nanohole Optical Tweezers, Nano Letters 2016, 16, 4, 2639-2643

40. A. S. Dimitrov, K. Nagayama, Continuous Convective Assembling of Fine Particles into Two-Dimensional Arrays on Solid Surfaces, Langmuir 1996, 12, 5, 1303-1311. 